Human and Animal Health

Vol.59: e16160208, January-December 2016 http://dx.doi.org/10.1590/1678-4324-2016160208 ISSN 1678-4324 Online Edition

BRAZILIAN ARCHIVES OF BIOLOGY AND TECHNOLOGY

AN INTERNATIONAL JOURNAL

\title{
The immunogenicity and anti-tumor effects of a lung cancer DNA vaccine harboring a MUC-1 and GM-CSF fusion gene
}

\author{
Zhen Yang ${ }^{1 *}$; DanDan Jiang ${ }^{1}$; Qiang Zhu1; BinBin Xiao'; Liang An Chen'. \\ ${ }^{1}$ Chinese PLA General Hospital - Department of Respiratory Disease, Beijing, China.
}

\begin{abstract}
DNA vaccines have been shown to be an effective approach to induce antigen-specific cellular and humoral immunity. However, the inability of DNA vaccines to elicit strong immune responses in clinical trials limits the application of DNA vaccines. Here, we developed a new DNA vaccine based on MUC1, which has been suggested as a potential target for lung cancer therapy, and we enhanced the potency of the DNA vaccine by including granulocytemacrophage colony-stimulating factor (GM-CSF) as an adjuvant. A series of DNA plasmids encoding MUC1, human GM-CSF and their conjugates were constructed and injected into female mice intramuscularly (i.m.). This action was followed by an electric pulse. The humoral and cellular immune responses after immunization were examined by ELISA and ELISPOT, respectively. To evaluate the therapeutic efficacy of the plasmids, a mouse model with a MUC1expressing tumor was designed. Mice vaccinated with the MUC1-GM-CSF plasmid generated the strongest MUC1specific humoral and cellular immune responses. Furthermore, these vaccinations inhibited the growth of MUC1expressing tumors and prolonged mouse survival. These observations emphasize the potential of GM-CSF as an adjuvant for DNA vaccines and of vaccines based on MUC1 and GM-CSF as a promising treatment for lung cancer.
\end{abstract}

Key words: MUC1 ; GM-CSF ; lung cancer; DNA vaccine

${ }^{1}$ Authors for correspondence: yztoogetyou@163.com 


\section{INTRODUCTION}

In recent years, immunotherapy technology has developed rapidly and has played an important role in the treatment of many diseases, especially in the treatment of cancer. This technology has great potential for further development and improving therapeutic effects of existing treatments [1]. DNA vaccines have received increasing attention due to their ability to induce both humoral and cellular immune responses. DNA vaccines can induce a strong CTL reaction in the treatment of cancer and chronic infectious diseases, and they have an advantage over other types of vaccines due to their strong CTL reactions [2].

DNA vaccines are made up of eukaryotic expression plasmids that carry an antigen of interest. When the DNA vaccine is injected into the body of an organism, the foreign target antigen is expressed in vivo, and this expression activates the body's immune system by inducing specific humoral and cellular immune responses [3, 4]. In recent years, rapid progress has been made in the development of DNA vaccines, particularly those that are used for the treatment of cancer and chronic infectious diseases [5]. Granulocyte macrophage colony stimulating factor (GM-CSF), which is produced by activated $\mathrm{T}$ cells and macrophages, has the ability to stimulate the proliferation, differentiation and maturation of hematopoietic progenitor cells. It also can activate dendritic cells, neutrophils, macrophages and other mononuclear cells, which have important immune regulatory functions in anti-tumor and anti-infection immunity [6].

In immune therapy, the selection of the target antigen also plays a vital role in the specificity and effectiveness of the treatment. MUC1 is a transmembrane glycoprotein normally expressed on the apical surface of ductal epithelia [7]. However, epithelial adenocarcinomas of several tissues, including breast, prostate, lung, colon, ovary and pancreas tissues, express an aberrant form of MUC1 [8, 9]. Active immunotherapy targeting tumors expressing MUC1 could have great value in cancer treatment. Moreover, a cancertargeting DNA vaccine encoding hMUC1 has been studied for its ability to target epithelial cancers that express high levels of MUC1. Furthermore, it has been shown that MUC1 DNA vaccination can inhibit MUC1 expression in tumor growth in a preclinical model.
In this study, a lung cancer DNA vaccine was constructed by cloning the MUC1 and GM-CSF genes linked by Furin-2A (F2A) [10] into pVAX1. The fusion construct was designed to improve the immunogenicity of a lung cancer DNA vaccine by using GM-CSF as an immune adjuvant, and this study explores the resulting anti-tumor activity.

\section{MATERIAL AND METHODS}

\section{Plasmid DNA constructs and preparation}

Based on the coding regions of MUC1 (GenBank: X80761.1) and human GM-CSF (GenBank: M11220.1), we designed a MUC1 (coding gene 1768) and GM-CSF (coding gene 33-467) fusion gene. The MUC1 gene was connected to the GMCSF gene by Furin-2A (F2A) (AGGGCCAAGAGGGCACCGGTGAAACAGA CTTTGAATTTTGACCTTCTCAAGTTGGCGG GAGACGTCGAGTCCAACCCTGGGCCC).

Synthesis of the fusion gene was carried out by Invitrogen. The fusion gene was released from the cloning vector by EcoR I and Xba I and then cloned into the eukaryotic expression vector pVAX1. This resulted in the eukaryotic expression plasmid pVAX1-MUC1- F2A-GM-CSF, which was identified by double enzyme digestion. These plasmids were transiently transfected into COS7 cells, and flow cytometry and ELISA were performed to confirm the validity of these constructs. Plasmids for vaccination and in vitro transfection of mammalian cells were prepared with an EndoFree Plasmid Maxi kit (Tiangen Biochemical, Beijing).

\section{Mice and cell lines}

Female C57BL/6 mice (4-6 weeks old) were purchased from Beijing Weitong Lihua Experimental Animal Technology Co. Ltd. (Beijing, China). The mice were maintained in accordance with the Guide for the Care and Use of Laboratory Animals (National Institutes of Health Publication No. 85-23, Revised 1996). Experimental procedures conformed to international guidelines for the care and use of laboratory animals, and they were approved by the Animal Ethics Committee of Chinese PLA General Hospital. The Lewis carcinoma cell (LLC) line was purchased from the Shanghai Cell Institute (Shanghai, China). To generate a cell line that stably expressed human MUC-1, LLC cells were 
transfected with a plasmid carrying MUC-1 (pIRES-neo- MUC1), and then the transfectants were subjected to selection by treatment with G418. This cell line is hereafter referred to as LLC MUC1.

\section{Animal grouping and vaccination}

The experimental mice were randomly divided into 4 groups: the blank control group, the empty vector pVAX1 group, the pVAX1-MUC1 group and the pVAX1-MUC1-F2A-GM-CSF group. Each mouse was injected with $100 \mu \mathrm{g}$ of plasmid and subjected to electroporation. On the 7th day and the 14th day after the first immunization, the mice were given an immune boost. The mice were sacrificed fourteen days after the final immunization, and immunological tests were performed.

\section{Assaying serum for MUC1 antibodies}

To evaluate the humoral response in the mouse model, anti-MUC1 antibodies in the sera of immunized mice were detected by enzyme-linked immunosorbent assay (ELISA). Ninety-six-well microplates were coated with $10 \mu \mathrm{g} / \mathrm{ml}$ of a recombinant MUC1 antigen in a bicarbonate buffer and incubated at $4^{\circ} \mathrm{C}$ overnight. After blocking with $5 \%$ powdered milk in PBS containing Tween-20, mouse sera taken 14 days after the final immunization were serially diluted in PBS and incubated in the plates for $2 \mathrm{~h}$ at room temperature. After washing with PBS, the plates were incubated with a 1:1000 dilution of a horseradish peroxidase (HRP)-conjugated goat anti-mouse IgG antibody (Zhong Shan Jin Qiao Biological Technology, Beijing, China) at room temperature for $1 \mathrm{~h}$. The reaction was stopped with $2 \mathrm{M} \mathrm{H}_{2} \mathrm{SO}_{4}$, and the absorbance at $450 \mathrm{~nm}$ was measured using an ELISA plate reader.

\section{ELISPOT assay}

Fourteen days after the final vaccination, splenocytes from the control and vaccinated groups of mice were isolated. An IFN- $\gamma$ enzyme-linked immunospot (ELISPOT) assay was performed according to the manufacturer's protocol (Dakewe Biotech Ltd., Shenzhen, China). In brief, 96-well plates were coated with an anti-mouse interferon (IFN)- $\gamma$ monoclonal antibody (mAb) at $4^{\circ} \mathrm{C}$ overnight and then blocked for $1 \mathrm{~h}$ at $37^{\circ} \mathrm{C}$. Freshly isolated splenocytes $\left(4 \times 10^{5}\right.$ cells/well $)$ from each vaccinated mouse group were added to the wells and incubated with $10 \mu \mathrm{g} / \mathrm{ml}$ of a recombinant MUC1 protein for $36 \mathrm{~h}$. Each test condition was performed in triplicate. The spots were counted and analyzed with the ELISPOT Reader (AID, Germany).

\section{In vivo tumor treatment experiments}

To test the ability of the DNA vaccine to treat tumors in the mouse model, 4-6-week-old female C57BL/6 mice were injected subcutaneously in the right flank with $1.0 \times 10^{5}$ LLC - MUC- 1 cells. Three days after tumor cell injection, eight mice were vaccinated intramuscularly (i.m.) with $100 \mu \mathrm{g}$ of pVAX1, pVAX1-MUC1 or pVAX1-MUC1-F2AGM-CSF, and on the 7th and 14th days after the first immunization, the immune system was boosted. Tumor development was monitored in individual mice every 3 days, and tumor volume was calculated according to the following formula: $\mathrm{V}\left(\mathrm{mm}^{3}\right)=0.5 \times$ long diameters $\times$ short diameters ${ }^{2}$. Based upon tumor volume calculations, growth curves of the tumors were plotted. On the 40th day after tumor inoculation, mice were sacrificed by cervical dislocation. Tumors were removed and weighed, and the tumor growth inhibition rate was calculated. The formula for tumor inhibition rate was as follows: (average tumor weight in the control group-average tumor weight in the treatment group)/ average tumor weight of the control group $\times 100 \%$.

\section{RESULTS AND DISCUSSION}

\section{Expression of DNA vaccine plasmids in eukaryotic cells}

Expression of the DNA vaccine plasmids in COS7 cells were verified. Forty-eight $h$ after transfection with the recombinant plasmid pVAX1-MUC1F2A-GM-CSF, cells in 6-well plates were collected by trypsin digestion. Because of the nature of MUC1 expression in the cell membrane, the mouseanti-MUC1 antibody and FITC-labeled goat-antimouse IgG were incubated with these cells at room temperature for 60 minutes. Expression of the MUC1 antigen was detected by flow cytometry. MUC1 was found to be expressed by the cells (Figure 1A). Because GM-CSF is secreted, ELISA was performed on the culture supernatant to detect its expression. ELISA results showed that the average expression of GM-CSF in the pVAX1MUC1-F2A-GM-CSF transfected cultures was significantly higher than in the empty vector group (Figure 1B). 
A
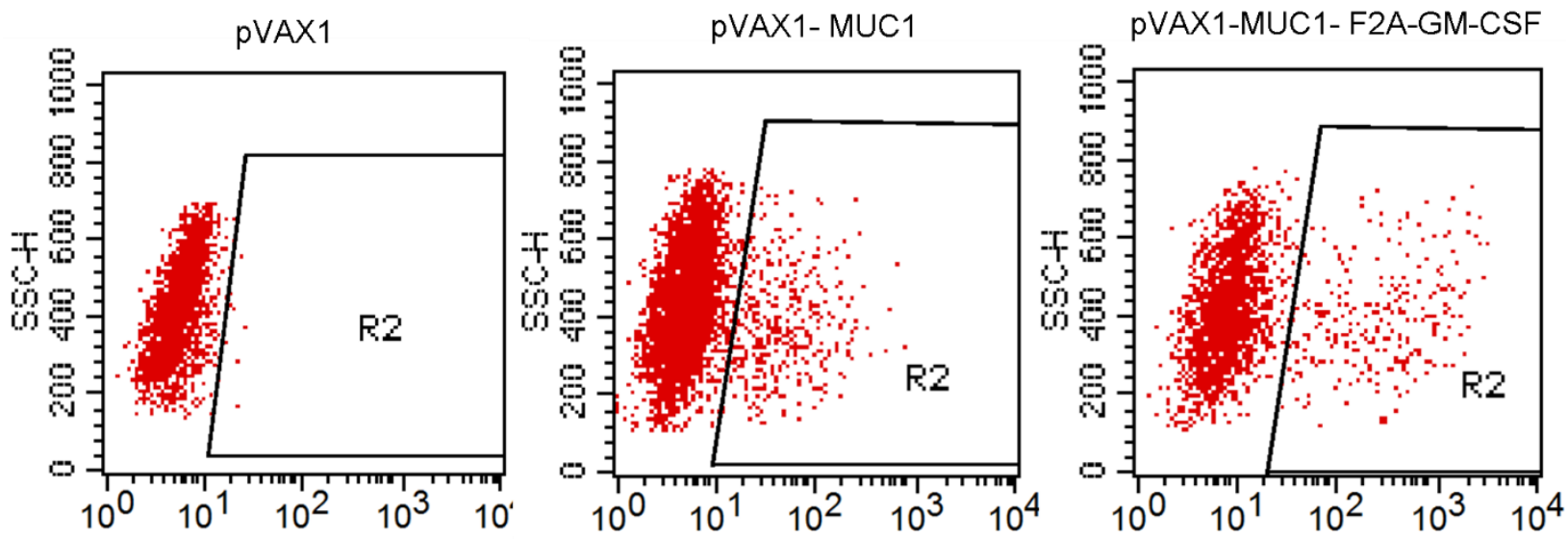

MUC1-FITC

B

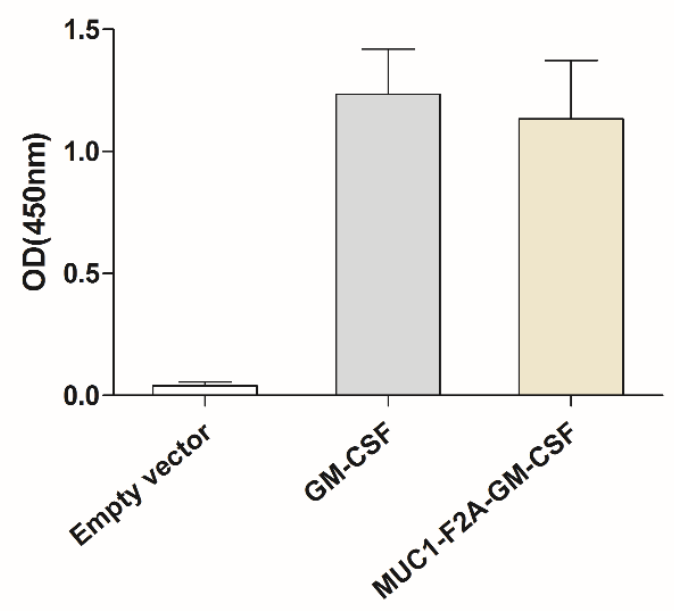

Figure 1. Expression of DNA vaccine plasmids. Flow cytometry and ELISA were used to examine MUC1 and GMCSF protein expression in COS7 cells, respectively. (A) Expression of MUC1 detected by flow cytometry. (B) Expression of GM-CSF detected by ELISA.

\section{Functional studies of the DNA vaccine}

Improving the immunogenicity of DNA vaccines by using molecular adjuvants is an important area of research. Cytokines are essential for the differentiation and maturation of immune cells and play a key role in the regulation of immune responses. It has been demonstrated that many cytokines alone have some anti-tumor effect (e.g. GM-CSF, IL-12 and IL-2), and some recombinant cytokines have been developed into an adjuvant in tumor treatments [11, 12]. Among these cytokines, GM-CSF is one of the better-studied molecules and has many roles in immune regulation. GM-CSF is produced by activated monocytes and $\mathrm{T}$ cells and stimulates the differentiation and maturation of hematopoietic progenitor cells; it can also activate macrophages, dendritic cells and neutrophils, and it plays a key role in cytokine networks. GM-CSF was used as a tumor therapy for the first time by Dranof and colleagues [13]; they found that GM-CSF produced a stronger and longer-lasting anti-tumor effect compared with their control group or other cytokines (IFN- $\gamma$, TNF- $\alpha$ and IL-2). To increase the immunogenicity and effectiveness of DNA vaccines, many studies [14] have fused GM-CSF with a specific antigen to make a molecular adjuvant. By doing this, humoral and cellular immune responses are significantly enhanced, tumor growth is inhibited, and the survival of experimental animals is improved.

To verify if the immunogenicity and anti-tumor effect can be enhanced through the expression of a 
fusion of GM-CSF, we designed and constructed a novel DNA vaccine that expresses MUC1 and GMCSF as an adjuvant. In mice vaccinated with pVAX1-MUC1-F2A-GM-CSF or pVAX1- MUC1 vaccines, the presence of anti-MUC1 antibodies was verified 2 weeks after the final immunization. As shown in Figure 2A, both the pVAX1-MUC1-

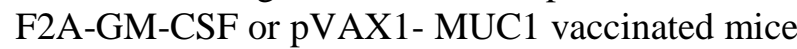
could induce specific anti-MUC1 antibodies. However, the recombinant plasmid pVAX1-

A

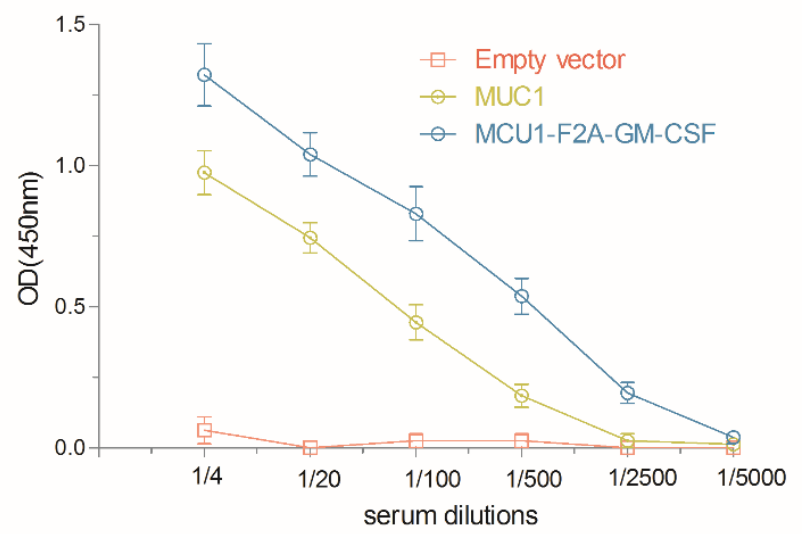

MUC1-F2A-GM-CSF induced antibody levels that were approximately 1.7 times than of the pVAX1MUC1 group (Figure 2B). The antibody titers of mice vaccinated with pVAX1-MUC1-F2A-GMCSF were significantly higher than those in other groups of mice $(\mathrm{P}<0.05)$. This experiment shows that GM-CSF significantly improves the antibody response induced by our DNA vaccine.

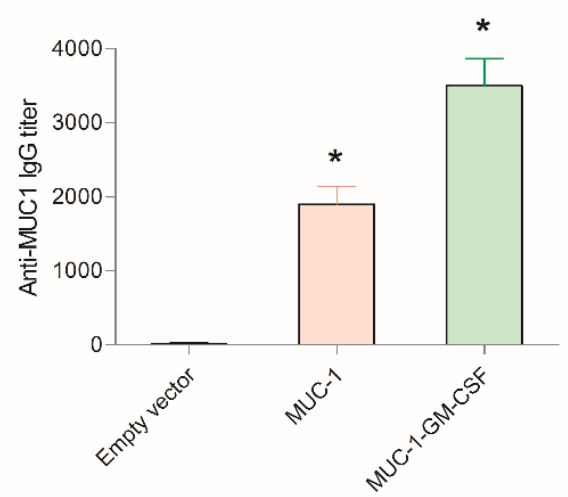

Figure 2. The MUC1-F2A-GM-CSF fused DNA vaccine induced anti-MUC1 antibody responses in mice. (A) Kinetics of the MUC1-specific antibody response. Groups of mice were immunized with the pVAX1, pVAX1-MUC1, or pVAX1-MUC1-GM-CSF plasmid. Anti-MUC1 antibodies were detected by ELISA. (B) Analysis of the MUC1specific antibody titer in the three groups of mice. The data are presented as the mean \pm S.D. There were five to eight mice per group.

Two weeks after the final immunization, an IFN- $\gamma$ ELISPOT assay of spleen lymphocytes was performed to detect the antigen-specific cellular immune response. As shown in Figure 3, MUC1stimulated IFN- $\gamma$ secretion was induced in the mice immunized with the pVAX1-MUC1 and pVAX1MUC1-F2A-GM-CSF vaccines. The spot numbers in the pVAX1-MUC1 and pVAX1-MUC1-F2AGM-CSF vaccinated groups were significantly higher than that in the empty vector $\mathrm{pVAX} 1$ group $(\mathrm{P}<0.05)$. Additionally, the spot number in the pVAX1-MUC1-F2A-GM-CSF group was higher than that in the pVAX1-MUC1 group $(\mathrm{P}<0.05)$ (Figure 3). These results indicate that IFN- $\gamma$ can significantly enhance the release of pVAX1MUC1-F2A-GM-CSF in the spleen cells of mice. Moreover, the antigen-induced release of IFN- $\gamma$ from spleen cells was significantly enhanced by the use of GM-CSF as an immune adjuvant. Together, these results from the ELISA and ELISPOT showed that the DNA vaccine induces both the humoral and cellular immune responses in mice and that the immune responses induced by the fusion gene are significantly higher than that of the antigen alone.

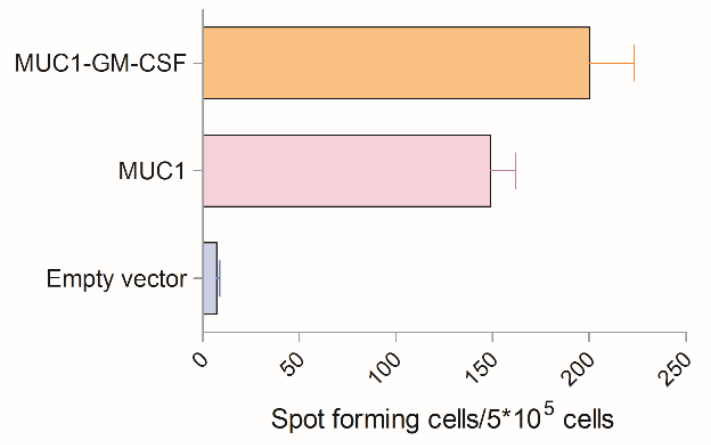

Figure 3. The MUC1-GM-CSF fused DNA vaccine induced MUC1-specific $\mathrm{T}$ cell responses in mice. Splenocytes from vaccinated mice were harvested 2 weeks after the final immunization. The MUC1-specific IFN- $\gamma$ ELISPOT level was measured as described in the Materials and methods section. The data are presented as the mean \pm S.D. There were five to eight mice per group. 
To study the effect of DNA vaccines on tumor growth, tumor-bearing mice were vaccinated with the DNA vaccine. The effect of immunotherapy was evaluated by measuring tumor size and mouse survival. Three days after tumor cell injection, mice were vaccinated three times with DNA vaccine plasmids, and the tumor volume was measured every 5 days. Tumor growth in mice treated with pVAX1-MUC1, pVAX1-GM-CSF or pVAX1MUC1-F2A-GM-CSF was significantly inhibited compared with that in the $\mathrm{pVAX} 1$ vector group (P $<0.05$ ) (Figure 4A). In addition, the pVAX1-

A

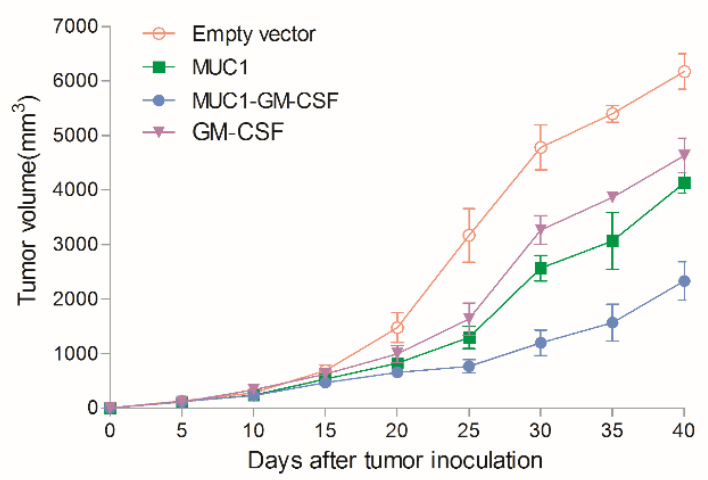

C
MUC1-F2A-GM-CSF vaccine inhibited tumor growth more effectively than the pVAX1-MUC1 and pVAX1-GM-CSF groups $(\mathrm{P}<0.05)$. We also measured tumor weight (Figure 4B) and calculated the tumor growth inhibition rate of the different groups of mice (Figure 4C) after vaccine treatment. Tumor weight and tumor growth inhibition rates in mice immunized with pVAX1-MUC1-F2A-GMCSF were significantly higher when compared with mice immunized with the pVAX1-MUC1 (P < $0.05)$ or $\mathrm{pVAX} 1$ vector $(\mathrm{P}<0.05)$ (Figure $4 \mathrm{C})$.

\section{B}

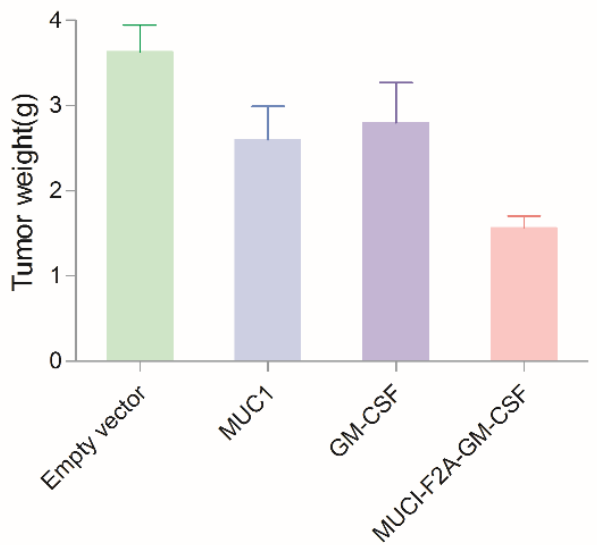

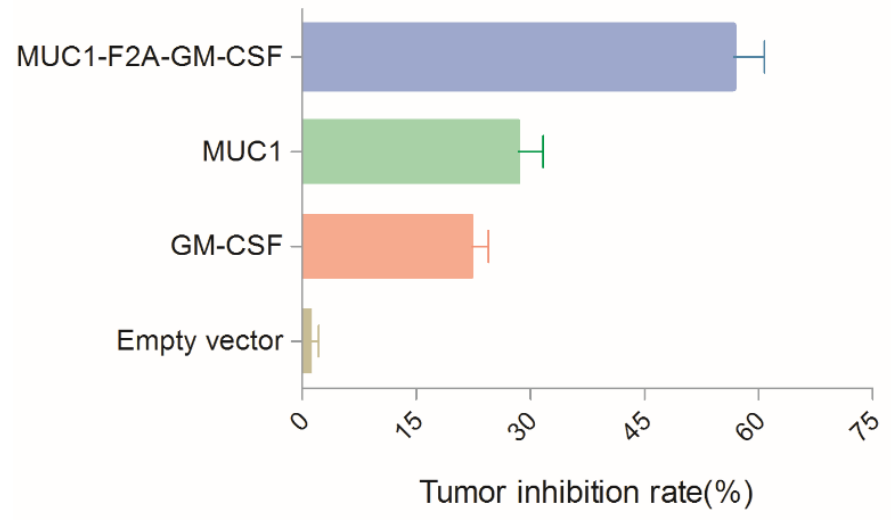

Figure 4. Anti-tumor efficacy of DNA vaccines in the tumor mouse model after immunization with pVAX1-MUC1F2A-GM-CSF. Three days after being challenged with tumor cells, the mice were immunized with the pVAX1, pVAX1-MUC1, or pVAX1-MUC1-GM-CSF plasmid. (A) Growth curve of MUC1 ${ }^{+}$LLC tumor. (B) and (C) Mean tumor weight and tumor inhibition rate. The data are presented as the mean \pm S.D. There were five to eight mice per group.

\section{CONCLUSIONS}

In this study, we demonstrated that vaccination with a fusion DNA vaccine encoding both MUC1 and 
GM-CSF enhanced the $\mathrm{B}$ and $\mathrm{T}$ cell responses through the use of GM-CSF as an adjuvant. Furthermore, this GM-CSF fusion DNA vaccine was able to inhibit tumor growth significantly more than the MUC1 antigen alone. Therefore, our study suggests that this model of increasing DNA vaccine immunogenicity through GM-CSF-antigen fusion is a promising anti-tumor therapy.

\section{ACKNOWLEDGMENT}

This work was supported by Youth development project of Military Medical Science and technology in China (No.13QNP175).

\section{CONFLICT OF INTEREST}

The authors declare no conflict of interest.

\section{REFERENCES}

1 Becht E, Giraldo NA, Dieu-Nosjean MC, SautèsFridman C, Fridman WH. Cancer immune contexture and immunotherapy. Curr Opin Immunol. 2016: 39;7-13.

2 Lee SH, Danishmalik SN, Sin JI. DNA vaccines, electroporation and their applications in cancer treatment. Hum Vaccin Immunother. 2015: 11(8); 1889-900.

3 Grunwald T, Ulbert S. Improvement of DNA vaccination by adjuvants and sophisticated delivery devices: vaccine-platforms for the battle against infectious diseases. Clin Exp Vaccine Res. 2015: 4(1); 1-10.

4 Guo C, Manjili MH, Subjeck JR, Sarkar D, Fisher PB, Wang XY. Therapeutic cancer vaccines: past, present, and future. Adv Cancer Res. 2013: 119; 421475.

5 Pol J, Bloy N, Obrist F, Eggermont A, Galon J, Hervé Fridman W, Cremer I, Zitvogel L, Kroemer G, Galluzzi L. Trial Watch: DNA vaccines for cancer therapy. Oncoimmunology. 2014: 3(1); e28185.

6 Kaufman HL, Ruby CE, Hughes T, Slingluff CL Jr. Current status of granulocyte-macrophage colonystimulating factor in the immunotherapy of melanoma.J Immunother Cancer. 2014: 13;2:11.

7 Pillai K, Pourgholami MH, Chua TC, Morris DL. MUC1 as a potential target in anticancer therapies. Am J Clin Oncol. 2015 :38(1) ;108-118

8 Deng J, Wang L, Chen H, Li L, Ma Y, Ni J, Li Y. The role of tumour-associated MUC1 in epithelial ovarian cancer metastasis and progression. Cancer Metastasis Rev. 2013: 32(3-4); 535-551.

9 Madsen CB, Wandall HH, Pedersen AE. Potential for novel MUC1 glycopeptide-specific antibody in passive cancer immunotherapy. Immunopharmacol Immunotoxicol. 2013: 35(6); 649-652.

10 Ho SC, Bardor M, Li B, Lee JJ, Song Z, Tong YW, Goh LT, Yang Y. Comparison of internal ribosome entry site (IRES) and Furin-2A (F2A) for monoclonal antibody expression level and quality in CHO cells. PLoS One. 2013:8(5); e63247.

11 Young PA, Morrison SL, Timmerman JM. Antibodycytokine fusion proteins for treatment of cancer: engineering cytokines for improved efficacy and safety.Semin Oncol. 2014: 41(5); 623-636.

12 Goshima F, Esaki S, Luo C, Kamakura M, Kimura H, Nishiyama Y. Oncolytic viral therapy with a combination of HF10, a herpes simplex virus type 1 variant and granulocyte-macrophage colonystimulating factor for murine ovarian cancer.Int $\mathbf{J}$ Cancer. 2014: 134 (12); 2865-2877.

13 Dranoff G, Jaffee E, Lazenby A, Golumbek P, Levitsky H, Brose K, Jackson V, Hamada H, Pardoll D, Mulligan RC. Vaccination with irradiated tumor cells engineered to secrete murine granulocyte-macrophage colony-stimulating factor stimulates potent, specific, and long-lasting anti-tumor immunity. Proc Natl Acad Sci U S A. 1993: 90(8); 3539-3543.

14 Li JW1, Gong JY, Li K, Liu Y, Ye QP, Liu X, Su ZX. Construction of a fusion expression plasmid containing the G250 gene and human granulocytemacrophage colony stimulating factor and its significance in renal cell carcinoma. Oncol Lett. 2011: 2(2); 343-347.

Received: January 15, 2016 Accepted: April 25, 2016 